\title{
Transtorno bipolar do humor e uso indevido de substâncias psicoativas Bipolar Disorder and Substance Abuse
}

\author{
Marcelo Ribeiro ${ }^{1}$ \\ RONALDO LARANJEIRA ${ }^{1}$ \\ Giuliana Cividanes ${ }^{2}$
}

\begin{abstract}
Resumo
O transtorno bipolar e o uso indevido de substâncias psicoativas são doenças com alto potencial de limitação de autonomia, tornando-se ainda mais sérios quando associados. Este artigo apresenta as evidências científicas disponíveis acerca da epidemiologia, etiologia, evolução clínica, diagnóstico e tratamento farmacoterápico e psicossocial da comorbidade transtorno bipolar do humor e o uso indevido de substâncias psicoativas.
\end{abstract}

Palavras-chave: Transtorno bipolar, abuso de substâncias, comorbidade, tratamento.

\begin{abstract}
The bipolar disorder and substance abuse are illness with highly autonomy limitation potential, becoming still more serious when associates. This article presents the available scientific evidences concerning the epidemiology, etiology, clinical evolution, diagnosis and pharmacological and psychosocial approaches designed for the bipolar disorder and substance abuse comorbidity.
\end{abstract}

Key words: Bipolar disorder, substance abuse, comorbidity, treatment.

\section{Introdução}

O uso indevido de substâncias psicoativas pelo paciente bipolar é extremamente comum e mais freqüente do que o observado na população geral (Kessler, 2004). Tal associação é capaz de alterar a expressão, o curso e o prognóstico de ambas as patologias (Levin e Hennesy, 2004; Krishnan, 2005), mesmo quando o consumo de álcool e/ou drogas é considerado de baixo risco ou moderado (Os et al., 2002; Shrier et al., 2003). A presença de outro transtorno psiquiátrico em dependentes químicos torna mais provável a procura desses por tratamento, fazendo com que as comorbidades 
sejam bastante recorrentes nos ambulatórios e enfermarias especializados (Grant, 1997; Hersh e ModestoLowe, 1998). Apesar da prevalência bem demonstrada, ainda há uma grande quantidade de lacunas, tais como a ausência de parâmetros diagnósticos confiáveis e a falta de metas terapêuticas apropriadas, que colocam o psiquiatra em situações de dúvida e indecisão. Este artigo apresenta as evidências científicas acerca da etiologia e da evolução clínica dessa comorbidade, seus critérios diagnósticos, bem como as estratégias farmacológicas e psicossociais disponíveis.

\section{Epidemiologia}

Os transtornos relacionados ao consumo de substâncias psicoativas estão entre as patologias psiquiátricas mais comuns. Em um estudo realizado em três capitais brasileiras (Brasília, São Paulo e Porto Alegre) no início dos anos noventa, o uso indevido de álcool foi detectado em quase $10 \%$ da população, e mais da metade desta estava desprovida de tratamento (Almeida-Filho et al., 1992). O I Levantamento Domiciliar sobre o Uso de Drogas no Brasil (2001) (Carlini et al., 2001) detectou que cerca de dois terços da população já consumiu álcool pelo menos uma vez na vida e outros $10 \%$ são dependentes da substância. Quanto ao uso de drogas ilícitas e prescritas, quase um quinto dos brasileiros o fez. Entre estudantes do ensino fundamental e médio, o consumo de álcool atinge $70 \%$ deles, enquanto o de outras substâncias, 25\% (Galduróz et al., 1997).

O transtorno bipolar do humor acomete cerca de 1\% da população geral brasileira (Almeida-Filho et al., 1992). Mesmo sendo pouco freqüente, seu impacto sobre a vida dos indivíduos e seus grupos de convívio se traduz em um sério problema de saúde pública. Além disso, o conceito da doença sofreu reformulações significativas nos últimos anos, ampliando-se o seu espectro (Angst, 2004). Desse modo, estudos mais recentes apontam para índices de prevalência até cinco vezes maiores, se comparados aos atuais (Akiskal, 1996). Apartir desses novos critérios, cerca da metade dos indivíduos com diagnóstico para depressão unipolar poderia ser considerada portadora de transtorno bipolar tipo II (Perugi e Akiskal, 2002). Isso significa que essa categoria de transtorno bipolar pode ser mais prevalente que a depressão maior, requerendo atenção redobrada por parte dos profissionais e órgãos de saúde (Angst, 2004).

O transtorno bipolar é a patologia do eixo I mais associada ao uso indevido de substâncias psicoativas. Os índices de comorbidade com o uso indevido de álcool atingem $60 \%$ a $85 \%$ desta população ao longo da vida (Regier et al., 1990; Vieta et al., 2001), enquanto o consumo de outras substâncias psicoativas (excluído o tabaco), de 20\% a 45\% (Strakowski e DelBello 2000; Krishnan, 2005). O uso de indevido de álcool e drogas parece ser mais prevalente no sexo masculino, apesar de não haver diferença com as mulheres no que concerne ao uso de algumas substâncias, como a cocaína (Cassidy, 2001). Além disso, o consumo pelas mulheres de substâncias psicoativas chega a ser de quatro a sete vezes mais intenso do que a média geral para o mesmo sexo (Hendrick et al., 2000).

Já entre os pacientes com problemas relacionados ao uso de álcool e drogas, um quarto apresenta algum transtorno do humor associado (Hasin e Nunes, 1998). Nos serviços especializados, a prevalência de dependentes químicos com depressão associada pode atingir $50 \%$ e com transtorno bipolar, de $20 \%$ a $30 \%$ (Grant, 1997; Hersh e Modesto-Lowe, 1998). Esses achados foram corroborados no Brasil por Cividanes (2001), que encontrou uma porcentagem de $22,4 \%$ de transtornos relacionados ao consumo de álcool entre 85 pacientes internados em dois hospitais psiquiátricos e um serviço ambulatorial. Por outro lado, Menezes e Ratto (2004) observaram psicóticos (incluindo o transtorno bipolar do humor) em diversos ambientes de tratamento em São Paulo e encontraram uma baixa porcentagem de uso nocivo $(4,2 \%)$ e dependência $(3,1 \%)$ deálcool. Quanto ao uso indevido de outras drogas, $8,3 \%$ dos participantes referiram algum consumo nos últimos 12 meses, sendo a maconha $(5,2 \%)$, os benzodiazepínicos $(4,1 \%)$ e a cocaína $(2,6 \%)$, os mais consumidos.

Antecedentes familiares de uso indevido de álcool e drogas são muito comuns nos pacientes acometidos pela comorbidade em questão (Nolen et al., 2004). A prevalência é ainda maior quando, além do transtorno bipolar e do uso de substâncias psicoativas, um transtorno de conduta é diagnosticado (Biederman et al., 2000).

\section{Hipóteses etiológicas}

A razão para os altos índices de uso indevido de substâncias em indivíduos com transtorno bipolar do humor é desconhecida. Atualmente, reconhece-se que as relações etiológicas entre o consumo de álcool e drogas e os transtornos afetivos são complexas, heterogêneas, bidirecionais e variáveis ao longo do tempo. Algumas hipóteses já foram aventadas (Quadro 1). Apesar de observadas em algumas situações, nenhuma destas é aplicável a todos os casos, podendo também aparecer associadas em sinergismo (Brady 1999; McQueen e Young, 2001).

Sintomas depressivos podem predispor o uso de substâncias químicas (hipótese da automedicação). Podem também resultar de dificuldades socioeconômicas (desemprego, separações afetivas etc.) decorrentes de um consumo mal-adaptado (hipótese socioeconômica) ou serem decorrentes de alterações neuroquímicas (transitórias ou persistentes) produzidas pelo uso crônico ou pela síndrome de abstinência (hipótese neurotóxica). Por fim, é possível que sejam patologias independentes ocorrendo coincidentemente num mesmo indivíduo (hipótese genética) (Merikangas e Stevens, 1998; Strakowski e DelBello, 2000). 
Quadro 1. Hipóteses etiológicas para a ocorrência de comorbidades entre o consumo de substâncias químicas e os transtornos afetivos.

- Sintomas do humor predispõem o uso de substâncias (hipótese da automedicação)

- 0 uso de substâncias químicas leva à comorbidade (hipótese da toxicidade)

- As repercussões socioeconômicas do uso levam à comorbidade

- Ambos os transtornos têm uma causa comum

- Ambos são fatores independentes

\section{Evolução clínica}

Algumas evidências apontam que o transtorno bipolar geralmente antecede o uso indevido de substâncias (Kupka et al., 2001; Kessler, 2004). Ainda assim, o uso de álcool e drogas pode contribuir para o surgimento da primeira crise (depressão ou mania) em certos grupos de pacientes, habitualmente em idade mais precoce do que a observada em pacientes bipolares não-usuários (Strakowski et al., 2000; Levin e Hennesy, 2004). Apesar de tais achados ainda serem escassos e carecerem de melhor entendimento (Krishnan, 2005), o transtorno bipolar é considerado fator de risco para o uso indevido de substâncias (Biederman et al., 1997) e o uso de álcool e drogas, um agente complicador e sinal de prognóstico reservado, tanto para a evolução do transtorno bipolar quanto para a resposta ao seu tratamento (Feinman e Dunner, 1996; Drake et al., 2004; Kessler, 2004).

$\mathrm{O}$ risco para o desenvolvimento dessa comorbidade é ainda maior quando o transtorno bipolar se inicia precocemente (Tohen et al., 1998) ou vem acompanhado ou precedido por transtornos de conduta (Levin e Hennesy, 2004) e impulsividade (Swann et al., 2004). Carlson et al. (1998) examinaram dados do Epidemiological Catchment Area (ECA) para determinar a ocorrência de transtorno bipolar, transtornos de conduta e abuso de substância em 132 indivíduos que preenchiam os critérios para o transtorno bipolar. Eles detectaram que aqueles com transtorno de conduta na infância tinham maior probabilidade de desenvolvimento do uso indevido de substâncias se comparados aos bipolares sem problemas de comportamento durante a infância. Há ainda a possibilidade de os transtornos de conduta e comportamentos disruptivos infantis serem, na realidade, sintomas de mania na infância, uma categoria nosológica que recebeu pouca atenção dos profissionais até recentemente, reforçando o elo causal entre o transtorno bipolar e o uso indevido de substâncias psicoativas na idade adulta (Faedda et al., 2004; Levin e Hennesy, 2004; Lima, 2004).

Se a relação de causalidade é ainda entremeada por inúmeras lacunas e incertezas, o papel do consumo de substâncias como fator de piora na evolução do transtorno bipolar do humor é bem estabelecido e consensual (Rush, 2003). Nolen et al. (2004) acompanharam, por um ano, 258 pacientes bipolares e constataram que a gravidade dos sintomas observados estava diretamente relacionada ao consumo de substâncias pelos pacientes, antecedentes de dependência de álcool e drogas pelos pais e baixo nível ocupacional. Segundo Krishnan (2005), as complicações do consumo de substâncias sobre o transtorno bipolar incluem maior incidência de episódios mistos e ciclagem rápida, aumento do tempo de remissão das crises, maior incidência de complicações clínicas e aumento dos índices de tentativa de suicídio e suicídio entre estes indivíduos. Outros achados apontam para uma estreita relação entre o consumo de álcool e drogas e o aumento da intensidade dos sintomas de depressão e mania, do fracasso na resposta às abordagens terapêuticas (medicamentosas e psicossociais) e do número e duração das internações (Salloum et al., 2002; Drake et al., 2004; Levin e Hennesy, 2004).

\section{Álcool}

O uso indevido de álcool é a comorbidade mais associada ao transtorno bipolar do humor (Vieta et al., 2001), sendo essa condição até cinco vezes mais prevalente entre os pacientes bipolares do que na população geral (Kessler, 2004). O consumo de álcool entre pacientes bipolares aumenta o risco de crises (especialmente a depressão), internações e tentativas de suicídio (Cividanes, 2001). Salloum et al. (2002) conduziram um estudo com 256 pacientes em mania internados para tratamento. Os pacientes que também possuíam diagnóstico para uso nocivo/dependência de álcool apresentaram mais sintomas de mania, maior labilidade do humor, impulsividade e episódios de violência do que aqueles sem uso atual de álcool. Slama et al. (2004) estudaram 307 pacientes bipolares em tratamento ambulatorial e constataram que a presença de uso indevido de álcool aumentou o risco de suicídio da amostra.

\section{Cocaína}

O consumo de cocaína chega a acometer um terço dos pacientes bipolares, aumentando ainda mais quando o transtorno bipolar está associado a um transtorno de ansiedade (pânico, fobia, TOC) (Goldberg et al., 
1999). A cocaína é utilizada mais comumente para manter ou potencializar o quadro de mania do que como "automedicação" dos sintomas depressivos (Crawford et al., 2003). A adesão é o principal problema dos usuários de cocaína (Goldberg et al., 1999; Crawford et al., 2003). Além disso, a associação entre o uso de cocaína e a ocorrência de problemas legais e transtornos de personalidade (Havassy e Arns, 1998) e a presença de uso concomitante de álcool e crack (Gossop et al., 2003) comprometem, per se, a evolução do curso da doença e do tratamento, representando mais um desafio para o especialista.

\section{Maconha}

Os efeitos deletérios da maconha estão relacionados à dose utilizada, à precocidade do início do consumo, às características de personalidade do usuário e à sua vulnerabilidade para complicações psiquiátricas (Os et al., 2002; Henquet et al., 2005). Arelação entre o consumo de maconha e o desenvolvimento de depressão maior foi documentada por alguns estudos. Bovasso (2001) entrevistou, 15 anos depois, 1.920 indivíduos que haviam participado, em 1980, do Epidemiological Catchment Area (ECA), na cidade de Baltimore. Entre os usuários de maconha sem diagnóstico inicial de depressão maior, o risco de aparecimento de sintomas depressivos entre as entrevistas foi quatro vezes maior, quando comparado ao grupo de não-usuários e sem transtorno psiquiátrico. Lynskey et al. (2004) acompanharam 277 pares de gêmeos, sendo apenas um deles dependente de maconha, e 311 pares de gêmeos, tendo apenas um deles iniciado o consumo antes dos 17 anos. Apenas entre os gêmeos dizigóticos, a dependência de maconha aumentou o risco de depressão maior. O risco de tentativa de suicídio foi três vezes maior entre os dependentes, sejam esses mono ou dizigóticos. O início precoce do consumo esteve associado ao risco de suicídio para ambos os tipos de gêmeos, mas não ao desenvolvimento de depressão. Tais achados sugerem que essa comorbidade possui componentes genéticos e ambientais.

No entanto, a relação entre o uso de maconha e o transtorno bipolar é menos conhecida. O consumo de maconha aumenta o risco, a intensidade e o tempo de duração de uma crise, geralmente polarizada para a mania (Strakowski et al., 2000). Rottanburg et al. (1982) observaram que um grupo de pacientes psicóticos com testes de urina positivos para maconha possuía crises marcadamente hipomaníacas, agitação psicomotora mais intensa, maior desorganização e duração mais prolongada da crise após o início do tratamento, se comparados com pacientes psicóticos livres da substância. Goldberg et al. (1999) constataram, que o consumo de maconha ou álcool em um grupo de pacientes bipolares internados aumentou o tempo de remissão do quadro, em comparação com bipolares não-usuários de substâncias psicoativas ou usuários de cocaína.

\section{Critérios diagnósticos}

O diagnóstico de ambas começa pela identificação da presença simultânea do transtorno bipolar do humor e transtornos relacionados ao consumo de substâncias psicoativas, a partir dos critérios da Classificação de Transtornos Mentais e de Comportamento da CID-10 / OMS ou do Manual Diagnóstico e Estatístico de Transtornos Mentais (DSM-IV / APA) (Quadro 2). Os critérios diagnósticos são objetivos e de fácil detecção pelo profissional capacitado. Adificuldade diagnóstica, no entanto, está na "atribuição causal acerca do quadro afetivo observado". A décima edição da Classificação Internacional das Doenças - CID-10 (OMS, 1993) não orienta os profissionais da saúde para essa questão, limitando-se a descrever os transtornos orgânicos do humor e os transtornos do humor propriamente ditos. Já o DSM-IV (APA, 1993) propõe algumas diretrizes para a identificação da natureza primária ou secundária dos transtornos mentais induzidos por substâncias (Quadro 3).

Um roteiro para a investigação de algumas hipóteses (Crome, 1999) pode ser útil para determinar a relação de causalidade entre as patologias observadas:

1. O consumo de substâncias (ainda que uma única vez) desencadeou o transtorno bipolar do humor.

2. A falta do consumo (síndrome de abstinência) causou ou exacerbou o transtorno bipolar do humor.

3. O transtorno bipolar do humor levou o indivíduo ao uso indevido de substâncias.

4. O transtorno bipolar do humor levou ao uso indevido de substâncias, que, por sua vez, repercutiu negativamente na evolução do primeiro.

Apesar de úteis na prática clínica diária, tais diretrizes estão longe da operacionalidade total. Conforme discutidas anteriormente, as relações de causa e efeito entre o transtorno bipolar e o uso indevido de substâncias ainda não estão totalmente esclarecidas e podem não ser as mesmas para todos os pacientes. Além disso, os sintomas relacionados à dependência de substâncias psicoativas e ao transtorno bipolar freqüentemente se sobrepõem (Brady et al., 1999).

Todas as substâncias psicoativas, especialmente os benzodiazepínicos, possuem "síndromes de abstinência protraídas", cursando com sintomas depressivos que excedem o período estabelecido pelo DSM-IV (Juergens e Cowley, 2003). Requerem, por isso, um julgamento clínico apurado no diagnóstico diferencial (Ashton, 1991). As substâncias químicas, per se, são capazes de induzir ou piorar síndromes depressivas ou maniformes, que remitem ou melhoram apenas com a abstinência, muitas vezes não requerendo tratamento medicamentoso posterior (Brunette et al., 2003; Crawford et al., 2003; Rush, 2003). Períodos de abstinência de substâncias como álcool, hipnóticos, opiáceos 
Quadro 2. Critérios da CID-10 e DSM-IV para dependência de substâncias.

CID-10

Um diagnóstico definitivo de dependência deve usualmente ser feito somente se três ou mais dos seguintes requisitos tenham sido experenciados ou exibidos em algum momento do ano anterior:

(a) um forte desejo ou senso de compulsão para consumir a substância;

(b) dificuldades em controlar o comportamento de consumir a substância em termos de seu início, término e níveis de consumo;

(c) um estado de abstinência fisiológico quando o uso da substância cessou ou foi reduzido, como evidenciado por: síndrome de abstinência para a substância ou o uso da mesma substância (ou de uma intimamente relacionada) com a intenção de aliviar ou evitar sintomas de abstinência;

(d) evidência de tolerância, de tal forma que doses crescentes da substância psicoativa são requeridas para alcançar efeitos originalmente produzidos por doses mais baixas:

(e) abandono progressivo de prazeres e interesses alternativos em favor do uso da substância psicoativa, aumento da quantidade de tempo necessária para se recuperar de seus efeitos;

(f) persistência no uso da substância, a despeito de evidência clara de conseqüências manifestamente nocivas (deve-se fazer esforços claros para determinar se o usuário estava realmente consciente da natureza e extensão do dano).

\section{DSM-IV}

Um padrão mal-adaptativo de uso de substância, levando a prejuízo clinicamente significativo, manifestado por três (ou mais) dos seguintes critérios, ocorrendo a qualquer momento no mesmo período de 12 meses:

(1) Tolerância, definida por qualquer um dos seguintes aspectos:

(a) uma necessidade de quantidades progressivamente maiores da substância para adquirir a intoxicação ou 0 efeito desejado;

(b) acentuada redução do efeito com o uso continuado da mesma quantidade de substância.

(2) Abstinência, manifestada por qualquer um dos seguintes aspectos:

(a) síndrome de abstinência característica para a substância;

(b) a mesma substância (ou uma substância estreitamente relacionada) é consumida para aliviar ou evitar sintomas de abstinência.

(3) A substância é freqüentemente consumida em maiores quantidades ou por um período mais longo do que o pretendido.

(4) Existe um desejo persistente ou esforços mal-sucedidos no sentido de reduzir ou controlar o uso da substância.

(5) Muito tempo é gasto em atividades necessárias para a obtenção da substância, na utilização da substância ou na recuperação de seus efeitos.

(6) Importantes atividades sociais, ocupacionais ou recreativas são abandonadas ou reduzidas em virtude do uso da substância.

(7) 0 uso da substância continua, apesar da consciência de ter um problema físico ou psicológico persistente ou recorrente que tende a ser causado ou exacerbado pela substância.

Quadro 3. Diretrizes diagnósticas do DSM-IV $(A P A, 1993)$ para a atribuição de um transtorno mental induzido por substância.

\section{Sinais de transtorno mental induzido por substância}

- Início durante a intoxicação ou abstinência, com melhora após a resolução destas.

- Presença de características atípicas do transtorno mental (por exemplo: primeiro episódio de mania após os 45 anos).

\section{Sinais de transtorno mental não-induzido por substância}

- Persistência dos sintomas a despeito da resolução do quadro de intoxicação ou abstinência por mais de quatro semanas.

- Sintomas surpreendentes em número e/ou intensidade em comparação aos observados nas síndromes de intoxicação ou abstinência.

- Episódios anteriores do transtorno mental livre do uso de substâncias.

Sinais de transtornos mentais mimetizados por substância

- Sinais e sintomas ligados às características fisiológicas e comportamentais da intoxicação ou da abstinência da substância, após a utilização de uma dose compatível ao quadro observado.

e estimulantes podem ser marcados por sintomas depressivos, tais como disforia, lentificação e alteração do sono, do apetite e da concentração (Brunette et al., 2003; Rush, 2003; Krishnan, 2005). Ao contrário dos sintomas depressivos, os quadros maniformes são induzidos principalmente pelo uso de estimulantes e alucinógenos (Halpern, 2003). Por fim, a combinação de agentes depressores e estimulantes em usuários pesados pode tornar ainda mais difícil a diferenciação entre os transtornos afetivos induzidos e não-induzidos por substâncias (Strakowski et al., 2000; Strakowski e DelBello, 2000; Crawford et al., 2003). 
Por outro lado, sintomas leves de mania, especialmente entre os cicladores rápidos e bipolares tipo II, são freqüentemente diagnosticados como sinais de recaída e fracasso dos pacientes em alcançar a abstinência ou como componentes da síndrome de abstinência da substância que utilizavam (Katzow et al., 2003). Sherwood et al. (2001) chamam a atenção para os altos índices de sintomas hipomaníacos entre pacientes de serviços ambulatoriais estadunidenses, ressaltando que estes, quando não diagnosticados, estão diretamente relacionados à baixa adesão e refratariedade ao tratamento instituído. Vieta et al. (2000) observaram que o transtorno bipolar tipo II é menos diagnosticado do que deveria, em decorrência da semelhança entre as suas sintomatologias e suas principais comorbidades, entre elas, o uso indevido de substâncias psicoativas.

Por fim, o transtorno bipolar do humor pode estar associado a outras patologias psiquiátricas, tais como déficit de atenção e hiperatividade e transtorno de personalidade borderline, ambos intimamente associados ao consumo de substâncias psicoativas (Sasson et al., 2003). Desse modo, a avaliação diagnóstica do psiquiatra deve ser cuidadosa, requerendo, na maior parte dos casos, algumas sessões com o paciente, familiares e grupos de convívio até a construção de um diagnóstico responsivo às estratégias de tratamento farmacológicas e psicossociais propostas.

\section{Tratamento}

O indivíduo com transtorno bipolar do humor, que faz uso indevido de substâncias psicoativas, requer acom- panhamento multidisciplinar especializado, de longa duração, preferencialmente em ambiente ambulatorial e supervisionado por uma única equipe profissional (NIDA, 2001; Department of Health, 2002).

Ainda que o tratamento apresente melhores resultados quando o transtorno bipolar e a dependência de substâncias psicoativas aparecem em suas formas puras, a abordagem dessa comorbidade pode ser bemsucedida. Drake et al. (2004) acompanharam por três anos o tratamento multidisciplinar de 51 pacientes com história de transtorno bipolar há mais de dez anos e dependência de substâncias psicoativas. Ao final do seguimento, observaram que a maioria estava abstinente e vivendo de maneira autônoma, com trabalho, contatos sociais com não-usuários de álcool e drogas e satisfeitos com sua qualidade de vida. No entanto, houve pouca ou nenhuma melhora dos sintomas psiquiátricos, que os autores consideraram "crônicos" e "arraigados".

A primeira meta terapêutica deve ser a remissão dos sintomas afetivos agudos e a obtenção de uma abstinência estável (McElroy, 2004). Isso aumenta a adesão ao tratamento e melhora o prognóstico de ambas as patologias (Department of Health, 2002). A gravidade da comorbidade não é per se um preditor de fracasso, mas o profissional deve possuir a sua dimensão exata (Figura 1), para que possa escolher a estratégia medicamentosa e o ambiente de tratamento mais adequados (Department of Health, 2002; Crawford et al., 2003).

\section{Farmacoterapia}

Há pouco conhecimento acerca de estratégias medicamentosas específicas para essa população. Segundo

\section{Gravidade do uso indevido}

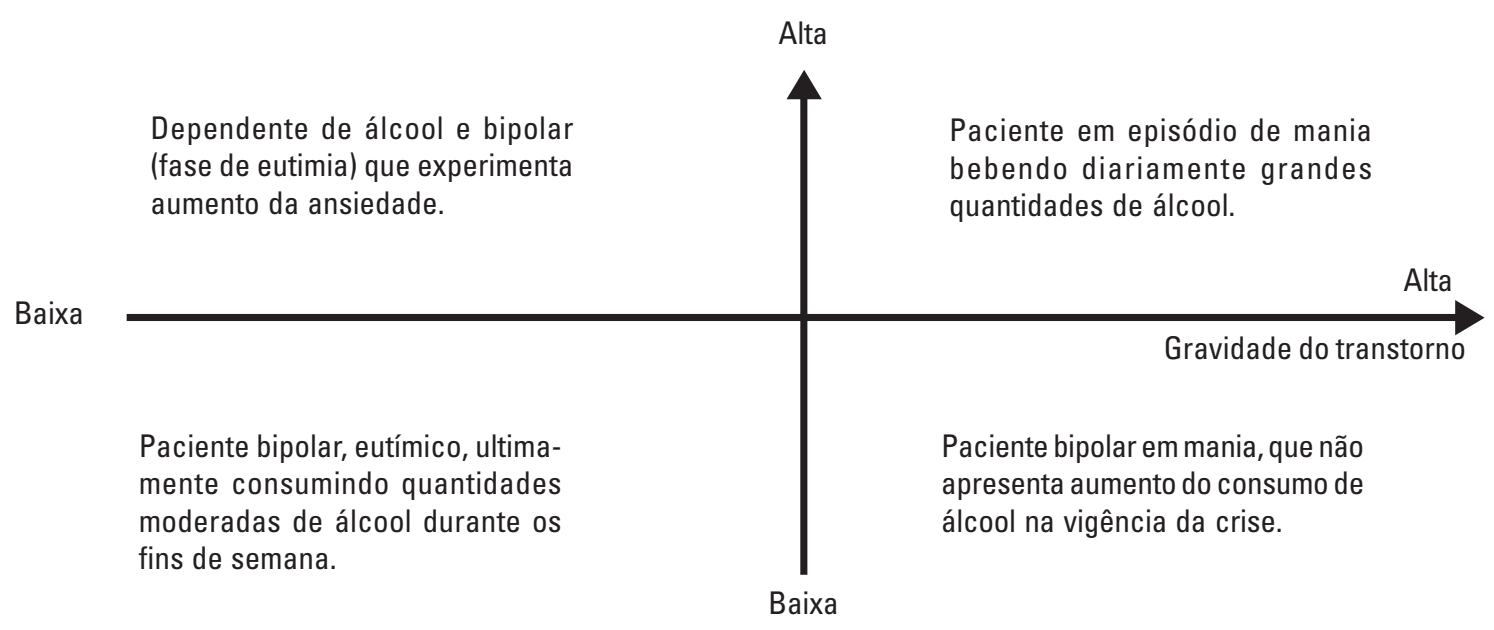

Figura 1. Aspectos relacionados à interação da gravidade do consumo de álcool e drogas em pacientes com transtorno bipolar do humor, com exemplos de quadros possíveis em cada condição. 
Kosten e Kosten (2004), a medicação ideal para o tratamento da comorbidade transtorno bipolar do humor e uso indevido de substâncias psicoativas deveria possuir as seguintes características: 1) remitir completamente as polarizações de humor (mania e depressão); 2) aliviar os sintomas de abstinência e fissura; 3) prevenir recaídas; 4) baixo potencial de abuso/ dependência; 5) via de administração e posologia de fácil manejo; 6) alta tolerabilidade pelo paciente. Os fármacos que melhor se aproximam desses critérios serão apresentados a seguir.

\section{Estabilizadores do humor}

Em pacientes com transtorno bipolar do humor e dependência de substâncias psicoativas, a utilização de qualquer tipo de estabilizador do humor contribui para a redução e melhora do padrão de consumo (Brown et al., 2001). O lítio, mesmo considerado a melhor opção terapêutica para o transtorno bipolar em sua forma pura (Macritchie et al., 2001), é significativamente menos eficaz entre bipolares com dependência de álcool e drogas (Kleindienst e Greil, 2003).

Estudos preliminares, em sua maioria abertos e não-controlados, têm sugerido o valproato e o divalproato de sódio como alternativas eficazes ao lítio, pois além de sua ação estabilizadora do humor, ambos têm-se mostrado capazes de diminuir a impulsividade, os comportamentos explosivos e a fissura em usuários de álcool e cocaína (Kosten e Kosten, 2004). Em um recente ensaio clínico, Salloum et al. (2005) acompanharam 59 pacientes bipolares tipo I e dependentes de álcool, randomizados em dois grupos. O grupo de pacientes tratado com lítio e divalproato reduziu significativamente seu padrão de consumo de álcool, se comparado ao grupo-placedo. O impacto sobre os sintomas afetivos foi semelhante para ambos os grupos. Parece haver uma relação entre a dose e a eficácia do divalproato sobre o uso indevido de drogas: Halikas et al. (2001), em um estudo aberto com usuários de cocaína tomando divalproato, encontraram menos testes de urina positivos para a substância entre aqueles com níveis séricos do medicamento acima de $50 \mu \mathrm{g} / \mathrm{ml}$.

Outra alternativa com potencial terapêutico é o topiramato: este estabilizador de ação gabaérgica temse mostrado eficaz na redução do craving e recaídas entre usuários de álcool (Kenna et al., 2004) e cocaína (Kampman et al., 2004; Rounsaville, 2004; Sofuoglu e Kosten, 2005). Por fim, a lamotrigina (Brown et al., 2003) e a gabapentina (Sokolski et al., 1999) mostraram-se eficazes na melhora dos sintomas depressivos e maniformes, do craving e da recaída em estudos abertos e não-controlados com usuários de cocaína. Não foram encontrados na literatura médica estudos acerca da eficácia do topiramato, da gabapentina e da lamotrigina em pacientes bipolares e usuários de substâncias psicoativas, tampouco estudos controlados e com maior índice amostral.

\section{Antipsicóticos}

Os antipsicóticos atípicos, especialmente entre pacientes bipolares tipo II e usuários de álcool e drogas, têmse mostrado eficazes como coadjuvantes dos estabilizadores do humor, tanto na remissão das crises de mania quanto no manejo da ansiedade (Albanese e Pies, 2004). Algumas questões acerca dessa classe medicamentosa ainda precisam de mais esclarecimentos, a fim de proporcionar mais segurança e precisão às estratégias farmacoterápicas dos especialistas. Por exemplo, alguns estudos aventam que a prescrição de neurolépticos poderia funcionar como um "gatilho" para o consumo de cocaína entre pacientes em mania que a utilizam para potencializar esse estado. Tal hipótese é corroborada por experimentos que demonstram aumento do consumo de estimulantes em animais tratados com neurolépticos típicos. Apesar de esse fenômeno ser menos recorrente entre os neurolépticos atípicos, há necessidade de mais estudos para elucidar melhor a questão (Brown et al., 2001).

\section{Antidepressivos}

O potencial terapêutico dos antidepressivos no tratamento da comorbidade depressão maior e uso indevido de álcool e drogas tem-se mostrado bastante desanimador. Nunes e Levin (2004) realizaram uma metanálise de artigos da literatura acerca do tema. Apenas artigos longitudinais, duplo-cegos e controlados foram incluídos. Os autores concluíram que os antidepressivos trazem apenas "benefícios modestos" para esses pacientes. Desse modo, nunca podem ser a única, tampouco a principal, abordagem instituída, devendo sempre estar associada a estratégias (farmacológicas e/ou psicossociais) direcionadas ao uso indevido de substâncias. Pettinati (2004) chegou a resultados semelhantes ao revisar a literatura acerca do uso de antidepressivos em dependentes de álcool com depressão maior. Apesar de contribuir para a remissão dos sintomas depressivos nesses indivíduos, tal melhora não teve impacto positivo sobre seu padrão de consumo de bebidas alcoólicas.

As evidências acerca do papel dos antidepressivos no tratamento da comorbidade em questão são escassas e carentes de mais embasamento científico. No entanto, parecem seguir a mesma tendência observada com a comorbidade depressão maior: são ineficazes quando desacompanhados de outros tratamentos (Salloum e Thase, 2000). Estudos abertos com inibidores seletivos da recaptação de serotonina são os mais recorrentes. Entre estes, a fluoxetina encontra melhor aceitação, em virtude da sua segurança, ação farmacológica sobre a impulsividade e baixa interação medicamentosa com substâncias psicoativas (Kosten e Kosten, 2004). Um estudo piloto com 13 usuários de cocaína deprimidos utilizando venlafaxina demonstrou que esta foi capaz de remitir os sintomas depressivos e reduzir o consumo de cocaína na maioria dos partici- 
pantes (McDowell et al., 2000). Um estudo maior e com grupo controle está sendo realizado no momento (Kosten e Kosten, 2004).

\section{Benzodiazepínicos}

Os benzodiazepínicos são prescritos com bastante freqüência (e eficácia comprovada) para os pacientes com transtornos do humor (Licht, 1998). O mesmo não pode ser comprovado quando o transtorno bipolar vem associado ao uso indevido de substâncias psicoativas: nesses casos, além de não ser eficaz no manejo dos sintomas de depressão, mania e ansiedade, aumentam consideravelmente o risco de dependência ao medicamento (Brunette et al., 2003). Desse modo, essa classe de substâncias deve ser utilizada com bastante reserva (ou mesmo evitada) para essa população (Roache et al., 1995; Johnson et al., 1998).

\section{Abordagens voltadas para a abstinência de substâncias psicoativas}

Qualquer abordagem famacoterápica para o tratamento da depressão ou mania perde eficácia diante do uso de drogas (Nunes e Levin, 2004). Desse modo, não se pode pensar no tratamento do transtorno bipolar do humor em usuários de substâncias psicoativas sem estruturar abordagens e atuar diretamente sobre o consumo de álcool e outras substâncias psicoativas. A abstinência continua a ser o melhor parâmetro para a execução de uma boa avaliação clínica e planejamento terapêutico do transtorno bipolar do humor (Brown et al., 2001; Nunes e Levin, 2004).

As abordagens motivacionais e cognitivo-comportamentais têm-se mostrado as mais promissoras (Department of Health, 2002; Carroll, 2004). Diversos programas específicos de tratamento têm aumentado a adesão, reduzido recaídas, remitido sintomas afetivos e melhorado a reinserção social entre os indivíduos bipolares com problemas relacionados ao consumo de álcool e drogas (Carroll, 2004).

\section{Programas de tratamento estruturados}

$\mathrm{O}$ tratamento das comorbidades deve ser planejado e estruturado longitudinalmente, com a participação do paciente e seus familiares. O Department of Health (2002) propõe um modelo de atenção multidisclipinar dividido em quatro etapas: engajamento, motivação para a mudança, tratamento ativo e prevenção da recaída com treinamento de habilidades sociais. Inicialmente, deve-se buscar o engajamento de todos para o tratamento, por meio de abordagens não-confrontativas e empáticas. Nesse período, a observação e o manejo de necessidades prementes, tais como complicações clínicas, resolução de conflitos interpessoais e melhora da qualidade de vida, podem ser mais importantes do que a abstinência imediata.

A partir do estabelecimento de um bom vínculo terapêutico, a motivação para a mudança deve ser trabalhada, visando a um estilo de vida livre de substâncias e mais compatível com a eutimia. Cabe nesse momento desenvolver atividades psicoeducativas com o paciente sobre o consumo de substâncias e suas repercussões na sua saúde física e mental, apresentar dados objetivos acerca da evolução do seu estado (exames do perfil hepático, testes neuropsicológicos etc.), promover um balanço dos prós e contras da abstinência e da permanência do uso, avaliar as barreiras e ameaças ao tratamento, levantar os problemas passados relacionados ao consumo de drogas e ao transtorno bipolar do humor e escolher a estratégia farmacoterápica mais adequada.

Superadas as etapas anteriores, começa o tratamento ativo. Ele deve ser implementado de maneira integrada, a partir de abordagens motivacionais e cognitivas, aconselhamento individual e em grupo, e com preocupação voltada ao suporte social. O período de tratamento é de longa duração. A quarta etapa complementa a terceira e consiste na instituição de técnicas de prevenção da recaída e habilidades sociais, a partir da identificação de fatores de risco de recaída e das necessidades do paciente.

\section{Conclusão}

Há uma série de lacunas acerca do entendimento etiológico do transtorno bipolar do humor associado ao uso indevido de substâncias psicoativas. Por outro lado, há uma grande variedade de estratégias medicamentosas e psicossociais em desenvolvimento, que ainda necessitam de estudos mais elaborados e por tempo mais prolongado. Entre as pesquisas farmacoterápicas em curso, merecem atenção especial aquelas acerca dos novos estabilizadores do humor, cujo potencial terapêutico parece contribuir tanto para a remissão dos sintomas afetivos, quanto para a melhora dos padrões de abstinência. A criação de programas estruturados de atenção, com participação da família e supervisionado por equipe multidisciplinar parece ser o caminho mais promissor. 
Referências bibliográficas

АкISKAL, H.S. The Prevalente Clinical Spectrum of Bipolar Disorders: Beyond DSM - IV. J Clin Psychopharmacol 16(Suppl. 1): 4-4, 1996.

Albanese, M.J.; Pies, R. - The Bipolar Patient with Comorbid Substance Use Disorder: Recognition and Management. CNS Drugs 18(9): 585-596, 2004.

Almeida Filho, N.; Mari, J.J.; Coutinho, E. et al. - Estudo Multicêntrico de Morbidade Psiquiátrica em Áreas Urbanas Brasileiras (Brasília, São Paulo, Porto Alegre). Rev ABP-APAL 14: 93-104, 1992.

American Psychiatric Association (APA) - Manual Diagnóstico e Estatístico de Transtornos Mentais DSM-IV. Artmed, Porto Alegre, 1993.

AngST, J. - Bipolar Disorder: A Seriously Underestimated Health Burden [editorial]. Eur Arch Psychiatry Clin Neurosci 254: 59-60, 2004.

Angst, J.; Gerber-Werder, R.; Zuberbühler, H.U. et al. - Is Bipolar I Disorder Heterogeneous? Eur Arch Psychiatry Clin Neurosci 254: 82-91, 2004.

Ashton, H. - Protracted with Drawal Syndromes from Benzodiazepines. J Subst Abuse Treat8 (1-2): 19-28, 1991.

Biederman, J.; Faraone, S; Wozniak, J. et al. - Parsing the Association between Bipolar, Conduct, and Substance Use Disorders: a Familial Risk Analysis. Biol Psychiatry 48: 1037-1044, 2000.

Biederman, J.; Wilens, T.; Mick E. et al. - Is ADHD a Risk for Psychoactive Substance Use Disorder? Findings from a Four Year Follow-Up Study. J Am Acad Child Adolesc Psychiatry 36: 21-29, 1997.

Bovasso, G.B. - Cannabis Abuse as a Risk Factor for Depressive Symptoms. Am J Psychiatry 158: 20362037, 2001.

Brady, K.T.; Sonne, S.C. - The Relationship between Substance Abuse and Bipolar Disorder. Psychiatr Clin N Am 22(3): 609-627, 1999.

Brown, E.S.; Nejtek, V.A.; Perantie, D.C. et al. - Lamotrigine in Patients with Bipolar Disorder and Cocaine Dependence. J Clin Psychiatry 64: 197-201, 2003.

Brown, E.S.; Suppes, T.; Adinoff, B. et al. - Drug Abuse and Bipolar Disorder: Comorbidity or Misdiagnosis? Bipolar Disord 65: 105-115, 2001.

BrunetTe, M.F.; Noordsy D.L.; XIE, H. et al. - Benzodiazepine Use and Abuse among Patients with Severe Mental Illness and Co-Occurring Substance Use Disorders. Psychiatr Serv 54: 1395-1401, 2003.

Carlini, E.A., Galduróz, J.C.F, Noto, A.R. et al. - I Levantamento Domiciliar sobre o Uso de Drogas Psicotrópicas no Brasil. CEBRID, São Paulo, 2001.

Carlson, G.A.; Bromet, E.J.; Jandorf, L. - Conduct Disorder and Mania: What Does It Mean in Adults. J Affect Disord 48: 199-205, 1998.

Carroll, K.M. - Behavioral Therapies for Co-occurring Substance Use and Mood Disorders. Biol Psychiatry 56(10): 778-784, 2004.
Cassidy, F.; Ahearn, A.P.; Carroll, J.B. - Substance abuse in bipolar disorder. Bipolar Disord 3: 181-188, 2001.

CIVIDANES, G.C. - Alcoolismo e Transtorno Bipolar do Humor: um Estudo de Comorbidade. São Paulo, 2001. Dissertação (Mestrado em Psiquiatria) - Escola Paulista de Medicina, Universidade de São Paulo.

Crawford, V; Crome, I.B.; Clancy, C. - Co-existing Problems of Mental Health and Substance Misuse (Dual Diagnosis): a Literature Review. Drugs Education Prev Policy 10 (Suppl): S10-74, 2003.

Crome, I.B. - Substance misuse and psychiatric comorbidity: towards improved service provision. Drugs: Education, Prevention and Policy 6(2): 151-174, 1999.

Department of Health - UK. Mental Health Policy Implementation Guide - Dual Diagnosis Good Practice Guide. Department of Health, London, 2002.

DrakE, R.E.; XIEA, H.; McHugo, G.J. et al. - Three-year Outcomes of Long-Term Patients with Co-occurring Bipolar and Substance Use Disorders. Biol Psychiatry 56(10): 749756, 2004.

Faedda, G.L., BaldessarinI, R.J.; Glovinsky, I.P. et al. - Pediatric Bipolar Disorder: Phenomenology and Course of Illness. Bipolar Disord 6: 305-313, 2004.

Feinman, J.A.; Dunner, D.L. - The Effect of Alcohol and Substance Abuse on the Course of Bipolar Affective Disorder. J Affective Disord 37: 43-49, 1996.

Galduróz, J.C.F.; Noto, A.R.; CarlinI, E.A. - IV Levantamento sobre o uso de drogas entre estudantes do ensino fundamental e médio de todas as capitais brasileiras. CEBRID, São Paulo, 1997.

Goldberg, J.F.; Garno, J.L.; Leon, A.C. et al. - A History of Substance Abuse Complicates Remission from Acute Mania in Bipolar Disorder. J Clin Psychiatry 60: 733740, 1999.

Gossop, M.; Marsden, J; Stewart, D. et al. - The National Treatment Outcome Research Study (NTORS): 4-5 year follow-up results. Addiction 98(3): 291-303, 2003.

Grant, B.F. - The Influence of Comorbid Major Depression and Substance Use Disorders on Alcohol and Drug Treatment: Results of a National Survey. In: National Institute on Drug Abuse (NIDA). Treatment of DrugDependent Individuals with Comorbid Mental Disorders. NIH, Rockville, 1997.

Halikas, J.A.; Halikas, B.A.; Center, V.L. et al. - A Pilot, open Clinical Study of Depakote in the Treatment of Cocaine Abuse. Hum Psychopharmacol 16: 257-264, 2001.

HalPeRn, J.H. - Hallucinogens: an Update. Cur Psychiatr Rep 5: 347-354, 2003.

HASIN, D.S.; Nunes, E.V. - Comorbidity of alcohol, drug and psychiatric disorders - epidemiology. In: Kranzler, H.R. \& Rounsaville, B.J. (eds.). Dual Diagnosis and Treatment: Substance Abuse and Comorbid Medical and Psychiatric Disorders. Marcel Dekker, New York, 1998. 
Havassy, B.E.; ArNs, P.G. - Relationship of Cocaine and other Substance Dependence to Well-Being of High-Risk Psychiatric Patients. Psychiatr Serv 49(7): 935-940, 1998.

Hendrick, V.; Altshuler, L.L.; Gituin, M.J. et al. - Gender and Bipolar Illness. J Clin Psychiatry 61: 393-396, 2000.

Henquet, C.; Krabbendam, L.; Spauwen, J. et al. - Prospective Cohort Study of Cannabis Use, Predisposition for Psychosis, and Psychotic Symptoms in Young People. BMJ 330(7481): 11, 2005.

Hersh, D.F.; Modesto-Lowe, V. - Drug Abuse and Mood Disorders. In: Kranzler H.R. \& Rounsaville, B.J. (eds.). Dual Diagnosis and Treatment: Substance Abuse and Comorbid Medical and Psychiatric Disorders. Marcel Dekker, New York, 1998.

Johnson, B; Longo. L.P. - Considerations in the Physician's Decision to Prescribe Benzodiazepines to Patients with Addiction. Psychiatric Annals 28: 160-165, 1998.

Juergens, S.M.; Cowley, D.S. - The Pharmacology of Benzodiazepines and Other Sedative-Hypnotics, In: American Society of Addiction Medicine (ASAM). Principles of Addiction Medicine. 3rd ed. Washington DC: ASAM, 2003.

Kampman. K.M.; Pettinati, H; Lynch, K.G. et al. - A Pilot Trial of Topiramate for the Treatment of Cocaine Dependence. Drug Alcohol Depend 75(3): 233-240, 2004.

Katzow, J.J; Hsu, D.J.; GhaemI, SN. - The Bipolar Spectrum: a Clinical Perspective. Bipolar Disord 5: 436-442, 2003.

Kenna, G.A.; McGeary, JE; Swift, R.M. - Pharmacotherapy, pharmacogenomics, and the future of alcohol dependence treatment. Part 2. Am J Health Syst Pharm 61(22): 2380-2388, 2004.

KESSLER, R.C. - The epidemiology of dual diagnosis. Impact of Substance Abuse on the Diagnosis, Course, and Treatment of Mood Disorders. Biol Psychiatry 56(10): 738-748, 2004.

KLeindienst, N.; GreIL, W. - Lithium in the long-term treatment of bipolar disorders. Eur Arch Psychiatry Clin Neurosci 53(3): 120-125, 2003.

Kosten, T.R.; Kosten, T.A. New Medication Strategies for Comorbid Substance Use and Bipolar Affective Disorders. Biol Psychiatry 56(10): 771-777, 2004.

Krishnan, K.R. - Psychiatric and Medical Comorbidities of Bipolar Disorder. Psychosom Med 67: 1-8, 2005.

Kupka, R.W.; Nolen, W.A.; Altshuler, L.L. et al. The Stanley Foundation Bipolar Network. 2. Preliminary Summary of Demographics Course of IIIness and Response to New Treatments. Br J Psychiatry 41 (suppl 1): 77-83, 2001.

Levin, F.R.; Hennessy, G. - Bipolar Disorder and Substance Abuse. Biol Psychiatry 56(10):738-48, 2004.

LICHT, R.W. - Drug Treatment of Mania: a Critical Review. Acta Psychiatrica Scandinavica 97: 387-397, 1998.

LIMA. D. - Bipolar Disorder and Depression in Childhood and Adolescence. J Pediatr 80(Suppl 2): S11-S20, 2004.

LyNSKey, M.T.; GLowinski A.L.; Todorov, A.A. et al. - Major Depressive Disorder, Suicidal Ideation, and Suicide Attempt in Twins Discordant for Cannabis Dependence and Early-Onset Cannabis Use. Arch Gen Psychiatry 61(10): 1026-1032, 2004.
Macritchie, K.A.; Geddes, J.R.; Haslam, D.R. et al. - Valproic Acid, Valproate and Divalproex in Maintenance Treatment of Bipolar Disorder. Cochrane Database Syst Ver (3): CD003196, 2001.

McDowelL, D.M.; LEVIN, F.R.; SeRACINI, A.M. et al. - Venlafaxine Treatment of Cocaine Abusers with Depressive Disorders. Am J Drug Alcohol Abuse 26: 25-31, 2000.

McElroY, S.L. - Diagnosing and Treating Comorbid (Complicated) Bipolar Disorder. J Clin Psychiatry 65(Suppl 15): 35-44, 2004.

McQueEn, G.M.; Young, L.T. - Bipolar II Disorder: Symptoms, Course, and Response to Treatment. Psychiatr Serv 52: 358-361, 2001.

Menezes, P.R.; Ratro, L.R.C. - Prevalence of Substance Misuse among Individuals with Severe Mental IIIness in São Paulo. Soc Psychiatry Psychiatr Epidemiol 39: 212-217, 2004.

Merikangas, K.R.; Stevens, D.E. - Models of Transmission of Substance Use and Comorbidity Psychiatric Disorders. In: Kranzler, H.R. \& Rounsaville, B.J. (eds.). Dual Diagnosis and Treatment: Substance Abuse and Comorbid Medical and Psychiatric Disorders. Marcel Dekker, New York, 1998.

National Insitute on Drug Abuse (NIDA). Principles of Drug Addiction Treatment. Rockville: National Institute of Health, 2001.

Nolen, W.A.; Lukenbaugh, D.A.; Altshuler L.L. et al. - Correlates of 1-Year Prospective Outcome in Bipolar Disorder: Results from the Stanley Foundation Bipolar Network. Am J Psychiatry 161(8): 1447-1454, 2004.

NunEs, E.V.; LEVIN, F.R. - Treatment of Depression in Patients with Alcohol or Other Drug Dependence: a MetaAnalysis. JAMA 291 (15): 1887-1896, 2004.

Organização Mundial da Saúde (OMS). Classificação de Transtornos Mentais e de Comportamento da CID-10. Artmed, Porto Alegre, 1993.

Os, J.; BaK, M.; HansSen, M. - Cannabis Use and Psychois: a Longitudinal Population Based Study. Am J Epidemiol 156(4): 319-327, 2002.

Perugi, G.; AKISKAL, H.S. - The Soft Bipolar Spectrum Redefined: Focus on the Cyclothymic, Anxious-Sensitive, ImpulseDyscontrol, and Binge-Eating Connection in Bipolar II and Related Conditions. Psychiatr Clin North Am 25(4): 713-737, 2002.

Pettinati, H.M. - Antidepressant Treatment of Co-occurring Depression and Alcohol Dependence. Biol Psychiatry 56(10): 785-792, 2004.

Regier, D.A., Farmer, M.E.; Rae D.S. et al. - Comorbidity of Mental Disorders with Alcohol and Other Drugs of Abuse Results from the Epidemiologic Catchment Area (ECA) Study. JAMA 264(19): 2511-2518, 1990.

ROACHE, J.D.; WEISCH, R.A. - Findings from Self-Administration Research on the Addiction Potential of Benzodiazepines. Psychiatric Annals 25: 153-157, 1995.

Rottanburg, D.; Robins, A.H.; Ben-Arie, O. et al. - Cannabis Associated Psychosis with Hipomanic Features. Lancet 8312: 1364-1366, 1982.

RounsavilLe, B.J. - Treatment of Cocaine Dependence and Depression. Biol Psychiatry 56(10): 803-809, 2004. 
Rush, A.J. - Toward an Understanding of Bipolar Disorder and its Origin. J Clin Psychiatry 64 (Suppl 6): 4-8, 2003.

Salloum, I.M.; Corneluus, J.R.; Daley, D.C. et al. - Efficacy of Valproate Maintenance in Patients with Bipolar Disorder and Alcoholism: a Double-Blind PlaceboControlled Study. Arch Gen Psychiatry62(1): 37-45, 2005.

SAlloum, I.M.; CorneluUs, J.R.; MezzICH, J.E.; KirISCI, L.- Impact of concurrent alcohol misuse on symptom presentation of acute mania at initial evaluation. Bipolar Disord 4: 418-421, 2002.

Salloum, I.M.; Thase, M.E. - Impact of Substance Abuse on the Course and Treatment of Bipolar Disorder. Bipolar Disord 2: 269-280, 2000.

Sasson, Y.; Chopra, M.; Harrari, E. et al. - Bipolar Comorbidity: from Diagnostic Dilemmas to Therapeutic Challenge. Int J Neuropsychopharmacol 6(2): 139-144, 2003.

Sherwood, Brown E.; Suppes, T.; Adinoff, B. et al. - Drug abuse and bipolar disorder: comorbidity or misdiagnos? J Affect Disord 65(2): 105-15, 2001.

Shrier, L.A.; HarRIS, S.K.; KuRLANd, M. etal. Substance use problems and associated psychiatric symptoms among adolescents in primary care. Pediatrics 111(6 Pt 1):699-705, 2003.

Slama, F; Bellivier, F.; HenRy, C. et al. - Bipolar Patients with Suicidal Behavior: Toward the Identification of a Clinical Subgroup. J Clin Psychiatry 65(8): 1035-1039, 2004.
Sofuoglu, M.; Kosten, T.R. - Novel Approaches to the Treatment of Cocaine Addiction. CNS Drugs 19(1): 13-25, 2005.

SoKolski, K.N.; Green, C.; Maris, D.E. et al. - Gabapentin as an Adjunct to Standard Mood Stabilizers in Outpatients with Mixed Bipolar Symptomatology. Ann Clin Psychiatry 11: 217-222, 1999.

Strakowski, S.M.; DelBello, M.P.; Fleck, D.E. et al. The Impact of Substance Abuse on the Course of Bipolar Disorder. Biol Psychiatry 48:477-485, 2000.

Strakowski, S.M.; DelBello, M.P. - The Co-Occurence of Bipolar and Substance Use Disorders. Clinical Psychol Rev 20(2): 191-206, 2000.

Swann, A.C.; DougherTy, D.M.; PAZZAgLIA, P.J. et al. - Impulsivity: a Link between Bipolar Disorder and Substance Abuse. Bipolar Dis 6: 204-212, 2004.

Tohen, M.; Greenfield, S.F.; Weiss, R.D. et al. - The Effect of Comorbid Substance Abuse Disorders on the Course Bipolar Disorder: a Review. Harv Rev Psychiatry 6(3): 133-141, 1998.

Vieta, E.; Colom, F.; Corbella, B. et al. - Clinical Correlates of Psychiatric Comorbidity in Bipolar I Patients. Bipolar Disord 3: 253-258, 2001.

Vieta, E.; Colom, F.; Martinez-Aran, A. et al. - Bipolar II Disorder and Comorbidity. Compr Psychiatry 41(5): 339-343, 2000. 